\title{
Laser-Induced Transition between Nonlinear and Linear Resonant Behaviors of a Micromechanical Oscillator
}

\author{
Tao Yang* and Yves Bellouard \\ Galatea Lab, IMT/STI, Ecole Polytechnique Fédérale de Lausanne (EPFL), Rue de la Maladière 71b, \\ CH-2002 Neuchâtel, Switzerland
}

(Received 13 December 2016; revised manuscript received 6 April 2017; published 5 June 2017)

\begin{abstract}
We investigate both theoretically and experimentally a laser-based controlled tuning of the nonlinear behaviors of a single mechanical resonator. Thanks to localized three-dimensional modifications induced by femtosecond-laser irradiation, a Duffing-like oscillator is switched from a hardening resonance to a linear response and then to a softening resonance and exhibits a wide tunability of the resonant frequency and a remarkable increase of its linear dynamic range. The principles that underlie laser-tuned nonlinear oscillators are generic and simple, suggesting its wide applicability not only for micro- or nanooptomechanical systems but also as a generic framework for characterizing and understanding the physics of in-volume laser-affected zones.
\end{abstract}

DOI: 10.1103/PhysRevApplied.7.064002

\section{INTRODUCTION}

The nonlinear nature of micro- or nanomechanical oscillators leads to abundant studies of fundamental and applied sciences [1-5], including macroscopic quantum behaviors, coherent coupling, and frequency stabilization. An efficient mechanism to tune the nonlinearity and resonance frequency holds promise for various applications such as ultrasensitive sensing [6,7] and signal processing [8,9]. On one hand, as the size of resonators scales down, the nonlinear dynamics sets a fundamental limitation on the dynamic range for linear operation, leading to frequency instability and excessive noise degrading the performance. On the other hand, it may lead to unusual phenomena, such as strong modal coupling and macroscopic quantum behaviors.

Various methods, such as electrostatic [10], thermal [11], and mechanical [12] tuning, have been implemented to tune the nonlinear behaviors. However, precise manipulation or sophisticated control is involved, adding substantial complexity to the system. Here, we use a nonablative femtosecond laser to tailor the local properties of materials in order to tune the nonlinearity and resonance frequency of a micromechanical oscillator and to increase its dynamic range beyond the critical amplitude.

In this paper, a theoretical framework is set to show theoretically the way to tune the nonlinear response of an oscillator. For the sake of demonstration, we consider a double-clamped beam excited by a transverse distributed

\footnotetext{
*tao.yang@epfl.ch
}

Published by the American Physical Society under the terms of the Creative Commons Attribution 4.0 International license. Further distribution of this work must maintain attribution to the author(s) and the published article's title, journal citation, and DOI. load. Although this is a particular configuration, the theoretical analysis can be adapted and expanded to other types of nonlinear mechanical oscillators. This technique further suggests the possibility of broader and reversible tunability.

\section{THEORETICAL ANALYSIS}

The Duffing equation [13] taking the form $\ddot{z}(t)+$ $2 \zeta \dot{z}(t)+\alpha_{1} z(t)+\alpha_{3} z^{3}(t)=f \cos (\Omega t)$ offers a reasonably accurate model to capture the nonlinear behaviors of resonators [14]. In this equation, it is the cubic stiffness term $\alpha_{3}$ that determines the nonlinear response: hardening for $\alpha_{3}>0$, linear response for $\alpha_{3}=0$, and softening for $\alpha_{3}<0$. Because of the double-clamped configuration, hardening nonlinearity rapidly manifests as the beam is driven further out of the plane.

The time-variant displacement $y(x, t)$ of a doubleclamped beam is modeled using the Euler-Bernoulli beam theory [15]. Here, the model is further extended to take into account an initial beam curviness $v(x)$, which is commonly observed due to fabrication imperfection. Further demonstration will see its great influence on the nonlinear behaviors when it is comparable to the beam thickness. Furthermore, we consider both harmonic and static excitations on the beam. The oscillation equation writes

$E I \frac{\partial^{4} y}{\partial x^{4}}+\rho A \frac{\partial^{2} y}{\partial t^{2}}-T\left(\frac{d^{2} v}{d x^{2}}+\frac{\partial^{2} y}{\partial x^{2}}\right)=F_{\mathrm{dc}}+F_{\mathrm{ac}} \cos (\Omega t)$,

with $E$ the Young's modulus, $I$ the area moment of inertia, $\rho$ the density, $A$ the cross-sectional area, $F_{\mathrm{dc}}$ the static excitation, $F_{\text {ac }}$ the harmonic excitation, and $\Omega$ the excitation frequency. The total tension term $T$ is a sum of an initial tension $T_{0}$ that may be introduced during fabrication, a bending-induced tension inherent to the 
overconstrained nature of double-clamped beams, and, finally, a beam-curviness-induced tension like the initial tension that can be a by-product of the fabrication process. $T$ is expressed as

$$
T=T_{0}+\frac{E A}{L} \int_{0}^{L}\left[\frac{1}{2}\left(\frac{\partial y}{\partial x}\right)^{2}+\frac{\partial y}{\partial x} \frac{d v}{d x}\right] d x,
$$

with $L$ the beam length. Here, we pose $v(x)=e \sin (\pi x / L)$, meaning that the beam is initially bent by a maximum quantity $e$.

We approximate the solution $y(x, t)=u(t) w(x)$ using separation of variables near the fundamental resonance. Under such conditions, the first spatial mode taking the characteristic displacement at the center point of the beam can be expressed as $w(x)=1-\cos (2 \pi x / L)$. The Galerkin approach [16] is then used to arrange Eq. (1) into a Duffing-like equation describing the time-variant magnitude $u(t)$,

$$
\begin{gathered}
\ddot{u}(t)+k_{1} u(t)+k_{2} u^{2}(t)+k_{3} u^{3}(t)=b_{1}+b_{2} \cos (\Omega t), \\
k_{1}=\frac{4 \pi^{4}}{9} \frac{E}{\rho} \frac{h^{2}}{L^{4}}\left[1+\frac{1}{4 \pi^{2}} \frac{L^{2}}{E I} T_{0}+\frac{32}{3 \pi^{2}}\left(\frac{e}{h}\right)^{2}\right], \\
k_{2}=\frac{16 \pi^{3}}{3} \frac{E}{\rho} \frac{e}{L^{4}}, \\
k_{3}=\frac{4 \pi^{4}}{3} \frac{E}{\rho} \frac{1}{L^{4}},
\end{gathered}
$$

with $h$ the beam thickness. $u(t)$ is split into a static amplitude $S$ due to the static term $b_{1}$ and a harmonic amplitude $z(t)$ due to the harmonic term $b_{2} \cos (\Omega t)$ and writes $u(t)=z(t)+S$. This treatment leads to the governing equation for $z(t)$ :

$\ddot{z}(t)+2 \zeta \dot{z}(t)+\alpha_{1} z(t)+\alpha_{2} z^{2}(t)+\alpha_{3} z^{3}(t)=b_{2} \cos (\Omega t)$.

Here, $\quad \alpha_{1}=k_{1}+2 S k_{2}+3 S^{2} k_{3}, \quad \alpha_{2}=k_{2}+3 S k_{3}, \quad$ and $\alpha_{3}=k_{3} . \alpha_{2}$ and $\alpha_{3}$ are the quadratic and cubic nonlinear stiffness terms, respectively. The natural frequency $\omega_{0}$ is given as $\omega_{0}=\sqrt{\alpha_{1}}$. A phenomenological damping term $2 \zeta \dot{z}(t)$ is added to the left-hand side of Eq. (7) to account for the dissipative terms not taken in Eq. (3).

The bending-induced tension inherent to the doubleclamped configuration contributes to a positive cubic nonlinear term according to Eq. (6). The initial tension $T_{0}$ depending on its sign that can be set positive, null, or negative can increase or decrease the linear stiffness $\alpha_{1}$, suggesting a tunable resonance frequency. The initial beam curviness $v(x)$, on one hand, introduces a competitive (for negative $T_{0}$ ) or collaborative (for positive $T_{0}$ ) factor by increasing the linear stiffness shown in Eq. (4). On the other hand, it also introduces a quadratic nonlinear term shown in Eq. (5). Both $\alpha_{3}$ and $\alpha_{2}$ determine the nonlinear response of the system. The existence of static excitation and the corresponding static deflection correlate these parameters. Therefore, $T_{0}$ and $v(x)$ may fully control the dynamic behaviors.

The method of multiple scales [17] is used to determine a first approximation solution for Eq. (7),

$$
z(t)=a(t) \cos [\Omega t+\phi(t)]+O(\varepsilon)
$$

where the amplitude $a(t)$ and the phase $\phi(t)$ are time variant, and $\varepsilon$ is the bookkeeping parameter in the perturbation analysis. Introducing Eq. (8) into Eq. (7), the amplitude of a steady-state motion is determined by

$$
\zeta^{2}+\left[\left(\Omega-\omega_{0}\right)-\frac{3}{8} \frac{\alpha}{\omega_{0}} a^{2}\right]^{2}=\frac{b_{2}^{2}}{4 \omega_{0}^{2} a^{2}} .
$$

The overall nonlinear term $\alpha$ takes the form $\alpha=\alpha_{3}$ (10/9) $\alpha_{2}^{2} \omega_{0}^{-2}$ and determines the nonlinear types. When $\alpha_{3}$ is positive and dominating, $\alpha_{2}$ tends to decrease $\alpha$ while the resonance keeps a hardening effect. When $\alpha_{2}$ is large enough to change the sign of $\alpha$ to negative, the resonance switches to softening response. In particular, when $\alpha_{3}=(10 / 9) \alpha_{2}^{2} \omega_{0}^{-2}$, meaning that $\alpha_{2}$ and $\alpha_{3}$ cancel each other, the nonlinear terms have no effect on the response, and the system behaves like a linear resonator.

To appreciate the main factors in switching resonant behaviors, we first consider the case of negligible static excitation, for which an analytical expression for $\alpha$ is derived:

$\alpha=\frac{4 \pi^{4}}{3} \frac{E}{\rho} \frac{1}{L^{4}}\left\{1-160\left(\frac{e}{h}\right)^{2}\left[3 \pi^{2}+\frac{3 L^{2} T_{0}}{4 E I}+32\left(\frac{e}{h}\right)^{2}\right]^{-1}\right\}$.

Here, two design parameters $T_{0}$ and $e / h$ affect the sign of $\alpha$ and, in turn, have direct effects on the nonlinearity. This fact implies theoretically the possibility of switching resonant behaviors simply by adjusting these two parameters.

In the case of nonzero static excitation, as is often encountered in practice, the complete model is evaluated numerically. There, we consider a fixed $F_{\mathrm{dc}}$ so that the vibration amplitude does not exceed 2 times $h$, which corresponds to the typical experimental values that we discuss later. Figure 1 shows that both beam curviness and initial tension can change the sign of $\alpha . \omega_{0}$ increases as the curviness increases in Fig. 1(b). In Fig. 1(a), it goes through a local minimum as the axial compression decreases (here around 0.6). The position of that local minimum is determined by the direct excitation and the curviness. It is 


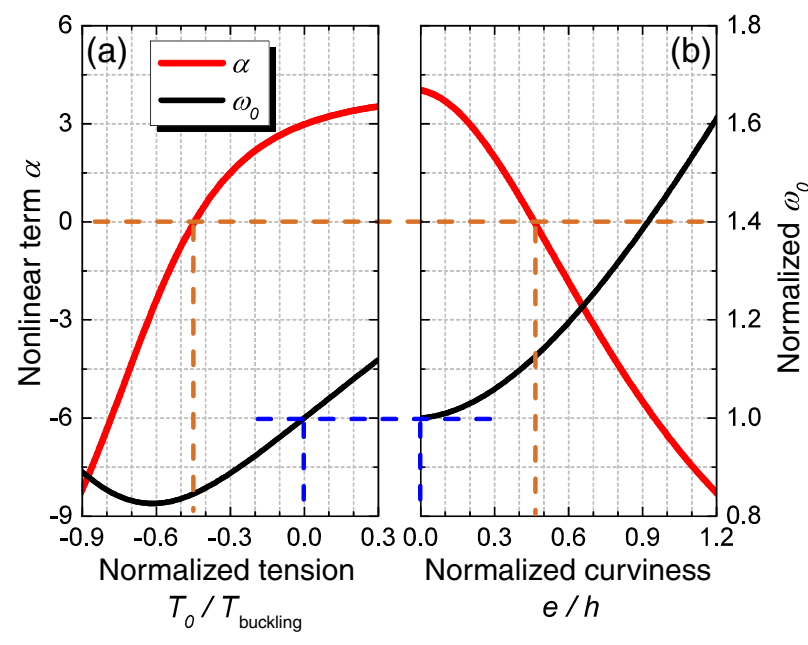

FIG. 1. (a) The effects of normalized tension $T_{0} / T_{\text {buckling }}$ on $\alpha$ and on normalized $\omega_{0}$. The normalized curviness is set to 0.2 . The normalized tension takes negative sign when it turns into compression and vice versa. $T_{\text {buckling }}$ denotes the absolute value of the critical buckling load. (b) The effects of normalized curviness $e / h$ on $\alpha$ and on normalized $\omega_{0} . T_{0}$ is set to 0 .

important to notice that $v(x), T_{0}$, and $F_{\mathrm{dc}}$ are inherently coupled.

\section{EXPERIMENTS AND RESULTS}

To investigate experimentally this model and the possibility of fully controlling the resonant behaviors of a single Duffing-like oscillator, we use a double-clamped beam manufactured by a process combining femtosecond laser and chemical etching. The femtosecond laser applied to transparent materials induces localized modifications in a well-confined three-dimensional manner due to nonlinear absorption phenomena [18]. This technique can be used for fabricating microdevices in particular and tailoring material properties in general. Here we use localized volume variations [19] and complex stress states [20] introduced by laser to vary $T_{0}$ and $v(x)$ in the beam.

The resonator design is depicted in Fig. 2(a). It consists of a double-clamped beam located below the chip surface and situated symmetrically between a pair of $\mathrm{V}$-shaped grooves fabricated by laser irradiation out of a single piece of silica substrate. The beam is excited using dieletrophoresis force [21] with details as follows [22]. First, a conductive coating partially covering the $\mathrm{V}$-shaped groove is used to create two electrodes, upon which an external driving voltage is applied. Second, a nonuniform electric field arises and induces a polarized dipolar moment on the dielectric beam. Because of the field nonuniformity, a net electrostatic force applied on the dipolar moment introduces an out-of-plane motion of the beam. In our experiments, a direct bias voltage $V_{\mathrm{dc}}$ is applied to excite the dipolar moment and to deflect the beam statically. A sinusoidal voltage $V_{\mathrm{ac}} \cos (\Omega t)$ is superimposed

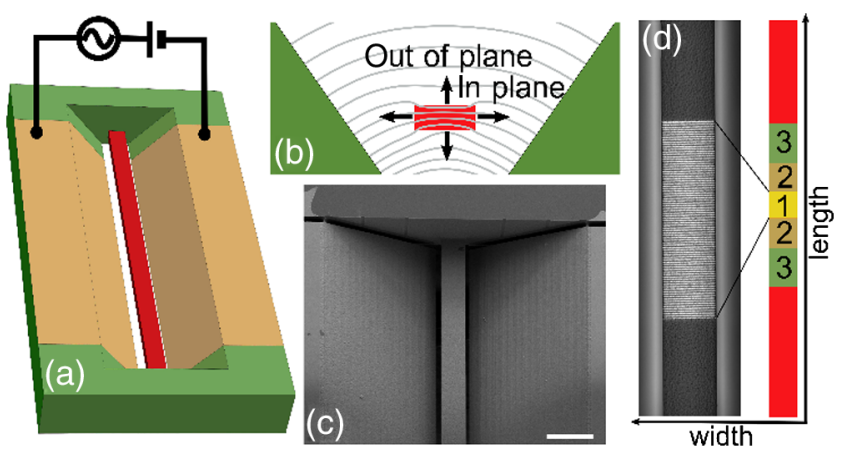

FIG. 2. (a) Illustration of a monolithic double-clamped resonator (in red) with its driving voltage source. (b) Schematic cross section of the beam and the $\mathrm{V}$-shaped groove boundaries. Finiteelement simulation is used to calculate the field distribution indicated by the electric field lines. The oscillation occurs out of the plane. (c) SEM image of the resonator showing one clamped end of the beam in a V-shaped groove. Scale bar is $200 \mu \mathrm{m}$. (d) Laser exposure strategy for tuning the nonlinear dynamics. Left optical microscopic image shows the top surface of the laserexposed volume. Right illustration summarizes the exposure sequences. After testing the dynamic response, a first irradiation (indicated by 1) is applied to the central volume of the beam over a certain length. After testing the response again, a second exposure (indicated by 2) consisting of two identical parts is conducted next to the first irradiated volume and so on. Exposure lengths vary depending on the beam lengths.

onto $V_{\mathrm{dc}}$ to drive the beam into resonance along the out-ofplane direction. The dielectrophoresis force is proportional to $\left[V_{\mathrm{dc}}+V_{\mathrm{ac}} \cos (\Omega t)\right]^{2}$. By neglecting higher-order harmonic components, it is expressed as $F \approx F_{\mathrm{dc}}+F_{\mathrm{ac}} \cos (\Omega t)$. A descriptive drawing of the actuation principle is shown in Fig. 2(b).

Figure 2(c) is a scanning-electron-microscopy (SEM) image showing the monolithic design of the resonator. Double-clamped beams of length 8 to $18 \mathrm{~mm}$ and crosssectional area (width $\times$ thickness) $100 \times 40 \mu \mathrm{m}^{2}$ are fabricated. A laser-displacement sensor is used to characterize the out-of-plane amplitude measured at the central location of the oscillating beams. The experiments are carried out under normal atmospheric pressure and at room temperature. We use a pulse energy of $200 \mathrm{~nJ}$ emitted from a 1030-nm laser with pulse duration of 280 fs to irradiate the central volume of double-clamped beams. Note that depending on the exposure conditions and pulse durations, some of the laser-induced material modifications can be erased by further treatment while maintaining the structural integrity of the device [23]. The laser scans transversely across the beam-width direction in a layer-by-layer manner along the beam-thickness direction defining a modified cross section. Then, it moves along the beam-length direction and repeats another cross-sectional scan. The pitches between the two consecutive lines along the beamlength and -thickness directions are 5 and $12 \mu \mathrm{m}$, respectively. These pitches are chosen such that no overlapping 


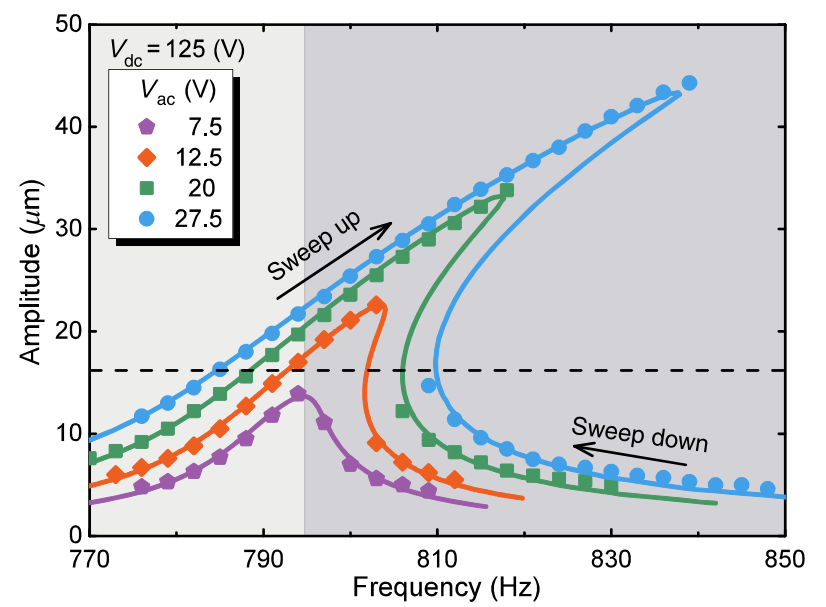

FIG. 3. Hardening nonlinearity of an 18-mm-long doubleclamped beam excited by dielectrophoresis force, with experimental data points comparing with theoretical fitted lines.

of the laser-affected zones takes place. Following the laser exposure sequence explained in Fig. 2(d), we can effectively change the sign of $\alpha$ and vary $\omega_{0}$ of a single oscillator.

A typical set of resonance curves displaying the intrinsic hardening nonlinearity before laser exposure is shown in Fig. 3. As the driving voltage increases, the frequency for peak amplitude is progressively pulled over to higher values. Hysteresis occurs as the response follows a steep fall to lower amplitudes when sweeping up to the peak frequency.

To tune $\alpha$ and $\omega_{0}$ and, therefore, to verify the prediction of the model developed, an 8-mm-long beam is locally tailored by the same laser. Figure 4(a) shows the initial hardening nonlinearity before the laser exposure. Figures 4(b)-4(d) further show the tuned nonlinear dynamics by a step-by-step laser exposure carried out on the same beam. After the first exposure, the hardening is "softened" and exhibits a decrease in resonance frequency. With more volume of the beam irradiated, the hardening switches to a linear response and with further irradiation, to softening. In all cases, the beam is driven at the same oscillation amplitude. The simulation also shows a transition of $\alpha$ from positive to negative, with an absolute minimum in the linear regime.

The ability to switch the nonlinear dynamics in Fig. 4 is due to the effective interaction between the femtosecond laser and material. The femtosecond laser creates porous structures [24] in nanogratings [25] inside laser-affected zones, and, as a consequence, it induces volume expansion and gives rise to a compressive stress along the beam, turning the initial tension into compression; that is, the sign of $T_{0}$ turns negative. With more laser exposure, the growing beam curviness due to imbalanced stress states induced on the top and bottom surfaces of the beam introduces $\alpha_{2}$ competing with $\alpha_{3}$. After the first exposure in Fig. 4(b), laser-induced volume expansion starts to create compressive stress in the beam, while a positive $\alpha_{3}$ due to midplane stretching is still dominating, corresponding to hardening dynamics with a reduced resonance frequency determined by Eq. (4). After the second exposure in Fig. 4(c), the hardening nonlinearity happens to be canceled by the softening effect due to laser-induced curviness $v(x)$ showing a linear dynamic response. With further exposure in Fig. 4(d), $\alpha$ turns negative, shifting the previous linear response to a softening one.

In addition to exhibiting linear and softening dynamics, Figs. 4(b)-4(d) all show a change in resonance frequency after exposure, which can also be understood based on the model derived earlier. Equation (4) shows that the resonance frequency is linearly proportional to the curviness $v(x)$, which grows by gradually increasing the irradiated volume, whereas laser-modified $T_{0}$ generally contributes to a decrease in resonance frequency according to $\omega_{0} \propto \sqrt{T_{0}}$. During the whole irradiation process, the laser-induced curviness comes into an overwhelming factor, tuning $\omega_{0}$ upwards. To verify the effective modification, we measure the beam curviness change due to laser irradiation. Typically, the center of the 8-mm-long beam bends by some $30 \mu \mathrm{m}$ after several exposures, which is comparable to the beam thickness.

A set of linear response curves after laser exposure with a comparison to its initial hardening is shown in Fig. 5.

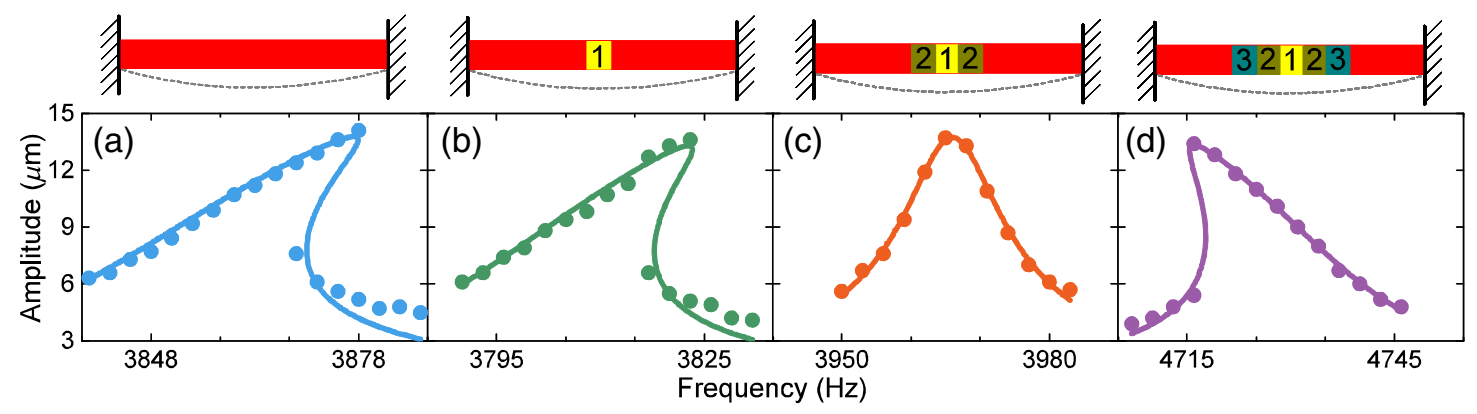

FIG. 4. Tuned dynamics of an 8-mm-long double-clamped-beam resonator by sequential femtosecond-laser exposure. Dots and solid lines are experimental data and theoretical prediction, respectively. (a) The intrinsic hardening response. (b) The first exposure takes place on the central $400 \mu \mathrm{m}$ length. (c) The second exposure of two 400- $\mu \mathrm{m}$ parts is carried out. (d) The third exposure of two 600- $\mu \mathrm{m}$ parts follows. 


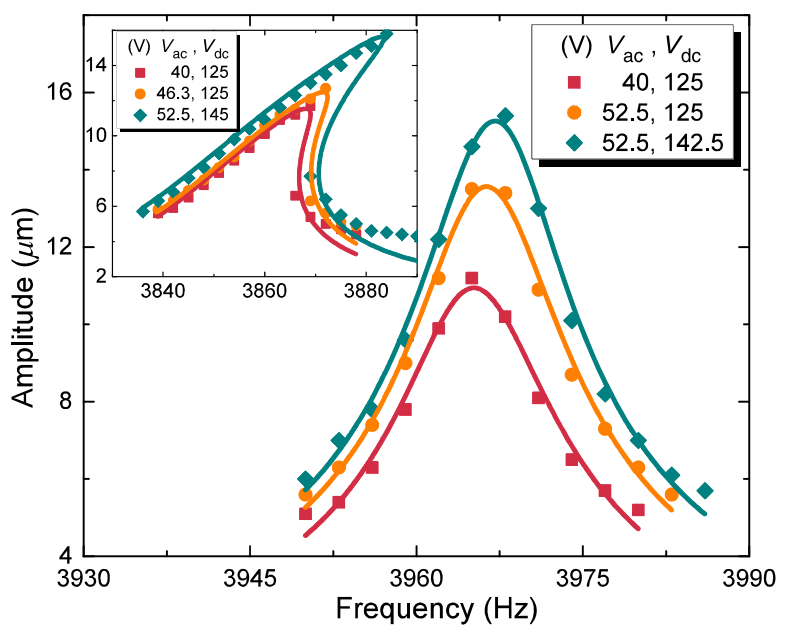

FIG. 5. A linear response of an 8-mm-long double-clampedbeam resonator tuned by laser irradiation. Measurements (points) are compared with the derived equations (lines). The dynamics is linear and, therefore, independent of the frequency-sweeping directions. Inset: Initial hardening nonlinearity at comparable amplitude before exposure.

This technique enables us to drive the oscillator beyond its critical amplitude, increase significantly the linear dynamic range [26] for linear operation, and enhance the signal-tonoise ratio and energy-handling capability.

In our analysis, we simplify some additional effects. The femtosecond laser may introduce a change in the Young's modulus of materials [27], for instance, due to structural porosity [28]. Furthermore, the laser-pulse energy is well below the ablation threshold for fused silica [29]; however, it can still cause localized ablation near the surfaces and change slightly the cross-sectional dimensions. These factors, to some extent, all play a role in tuning the nonlinear dynamics and cause discrepancies. Nevertheless, the model we introduce explains and agrees qualitatively with the tunable resonance and nonlinearity in experiments. A better match with experimental results requires in situ calibration and/or refining the model to include second-order effects.

\section{CONCLUSION}

In conclusion, we present a theoretical framework and its experimental validation for tuning the nonlinear response of a Duffing-like oscillator by exploiting femtosecond-laser-matter interaction. Most remarkable is the ability to tune the oscillator to display either softening or hardening or linear resonant behaviors and the capability to expand its linear dynamic range. Our methodology combining nonlinear mechanical resonators with femtosecond-laser-matter interaction possesses more potential. The femtosecond laser depending on the conditions can produce modifications of opposite properties, for instance, compressive and tensile stress. Some structural modifications, for example, the densification regime, can be erased by subsequent treatments [23]. Therefore, it suggests wide, rewritable, and reversible tunability and may pave the way for fundamental studies and applications, like, for instance, quantum effects in macroscopic resonators [2,30] and tunable devices. Finally, femtosecond-laser-tuned mechanical resonators can serve as a unique tool to investigate the complex thermoelastic properties of laser-modified structures, so far largely unknown, and that can have diverse morphologies ranging from self-organized nanogratings to densified zones with varying porosity levels [31], also including the possible presence of laser-induced polymorphic phases [32].

\section{ACKNOWLEDGMENTS}

This work is supported by the European Research Council (Grant No. ERC-2012-StG-307442). The authors acknowledge the sponsoring of Richemont International.

[1] H. J. R. Westra, M. Poot, H. S. J. van der Zant, and W. J. Venstra, Nonlinear Modal Interactions in ClampedClamped Mechanical Resonators, Phys. Rev. Lett. 105, 117205 (2010).

[2] P. Huang, J. Zhou, L. Zhang, D. Hou, S. Lin, W. Deng, C. Meng, C. Duan, C. Ju, X. Zheng, F. Xue, and J. Du, Generating giant and tunable nonlinearity in a macroscopic mechanical resonator from a single chemical bond, Nat. Commun. 7, 11517 (2016).

[3] R. Ohta, H. Okamoto, R. Hey, K. J. Friedland, and H. Yamaguchi, Optically induced strong intermodal coupling in mechanical resonators at room temperature, Appl. Phys. Lett. 107, 091906 (2015).

[4] E. Kenig, M. C. Cross, R. Lifshitz, R. B. Karabalin, L. G. Villanueva, M. H. Matheny, and M. L. Roukes, Passive Phase Noise Cancellation Scheme, Phys. Rev. Lett. 108, 264102 (2012).

[5] A. Castellanos-Gomez, H. B. Meerwaldt, W. J. Venstra, H. S. J. van der Zant, and G. A. Steele, Strong and tunable mode coupling in carbon nanotube resonators, Phys. Rev. B 86, 041402(R) (2012).

[6] V. Sazonova, Y. Yaish, H. Üstünel, D. Roundy, T. A. Arias, and P. L. McEuen, A tunable carbon nanotube electromechanical oscillator, Nature (London) 431, 284 (2004).

[7] H. Cho, M. Yu, A. F. Vakakis, L. A. Bergman, and D. M. McFarland, Tunable, broadband nonlinear nanomechanical resonator, Nano Lett. 10, 1793 (2010).

[8] R. B. Karabalin, R. Lifshitz, M. C. Cross, M. H. Matheny, S. C. Masmanidis, and M. L. Roukes, Signal Amplification by Sensitive Control of Bifurcation Topology, Phys. Rev. Lett. 106, 094102 (2011).

[9] S. S. Verbridge, D. F. Shapiro, H. G. Craighead, and J. M. Parpia, Macroscopic tuning of nanomechanics: Substrate bending for reversible control of frequency and quality factor of nanostring resonators, Nano Lett. 7, 1728 (2007).

[10] N. Kacem and S. Hentz, Bifurcation topology tuning of a mixed behavior in nonlinear micromechanical resonators, Appl. Phys. Lett. 95, 183104 (2009). 
[11] G. Jourdan, F. Comin, and J. Chevrier, Mechanical Mode Dependence of Bolometric Backaction in an Atomic Force Microscopy Microlever, Phys. Rev. Lett. 101, 133904 (2008).

[12] H. Cho, B. Jeong, M.-F. Yu, A. F. Vakakis, D. M. McFarland, and L.A. Bergman, Nonlinear hardening and softening resonances in micromechanical cantilever-nanotube systems originated from nanoscale geometric nonlinearities, Int. J. Solids Struct. 49, 2059 (2012).

[13] I. Kovacic and M. J. Brennan, The Duffing Equation: Nonlinear Oscillators and Their Behavior (Wiley, New York, 2011).

[14] K. Eom, H. S. Park, D. S. Yoon, and T. Kwon, Nanomechanical resonators and their applications in biological/ chemical detection: Nanomechanics principles, Phys. Rep. 503, 115 (2011).

[15] R. Lifshitz and M. C. Cross, Review of Nonlinear Dynamics and Complexity (Wiley-VCH Verlag, Weinheim, 2008), Vol. 1, pp. 1-52.

[16] A. H. Nayfeh and D. T. Mook, Nonlinear Oscillations, 1st ed. (Wiley, New York, 1979).

[17] S. H. Strogatz, Nonlinear Dynamics and Chaos-With Applications to Physics, Biology, Chemistry and Engineering (Addison-Wesley, Reading, MA, 1994).

[18] B. C. Stuart, M. D. Feit, A. M. Rubenchik, B. W. Shore, and M. D. Perry, Laser-Induced Damage in Dielectrics with Nanosecond to Subpicosecond Pulses, Phys. Rev. Lett. 74, 2248 (1995).

[19] A. Champion and Y. Bellouard, Direct volume variation measurements in fused silica specimens exposed to femtosecond laser, Opt. Mater. Express 2, 789 (2012).

[20] B. Poumellec, L. Sudrie, M. Franco, B. Prade, and A. Mysyrowicz, Femtosecond laser irradiation stress induced in pure silica, Opt. Express 11, 1070 (2003).

[21] H. A. Pohl, The motion and precipitation of suspensoids in divergent electric fields, J. Appl. Phys. 22, 869 (1951).
[22] T. Yang and Y. Bellouard, Monolithic transparent 3D dielectrophoretic micro-actuator fabricated by femtosecond laser, J. Micromech. Microeng. 25, 105009 (2015).

[23] E. Bricchi and P. G. Kazansky, Extraordinary stability of anisotropic femtosecond direct-written structures embedded in silica glass, Appl. Phys. Lett. 88, 111119 (2006).

[24] M. Lancry, B. Poumellec, J. Canning, K. Cook, J.-C. Poulin, and F. Brisset, Ultrafast nanoporous silica formation driven by femtosecond laser irradiation, Laser Photonics Rev. 7, 953 (2013).

[25] Y. Shimotsuma, P. G. Kazansky, J. Qiu, and K. Hirao, SelfOrganized Nanogratings in Glass Irradiated by Ultrashort Light Pulses, Phys. Rev. Lett. 91, 247405 (2003).

[26] H. W. Ch. Postma, I. Kozinsky, A. Husain, and M. L. Roukes, Dynamic range of nanotube- and nanowire-based electromechanical systems, Appl. Phys. Lett. 86, 223105 (2005).

[27] Y. Bellouard, T. Colomb, C. Depeursinge, M. Dugan, A. A. Said, and P. Bado, Nanoindentation and birefringence measurements on fused silica specimen exposed to lowenergy femtosecond pulses, Opt. Express 14, 8360 (2006).

[28] K. K. Phani and S. K. Niyogi, Young's modulus of porous brittle solids, J. Mater. Sci. 22, 257 (1987).

[29] M. Beresna, M. Gecevičius, and P. G. Kazansky, Ultrafast laser direct writing and nanostructuring in transparent materials, Adv. Opt. Photonics 6, 293 (2014).

[30] V. Peano and M. Thorwart, Macroscopic quantum effects in a strongly driven nanomechanical resonator, Phys. Rev. B 70, 235401 (2004).

[31] Y. Bellouard, A. Champion, B. McMillen, S. Mukherjee, R. R. Thomson, C. Pépin, P. Gillet, and Y. Cheng, Stressstate manipulation in fused silica via femtosecond laser irradiation, Optica 3, 1285 (2016).

[32] V. Oliveira, S. P. Sharma, P. Herrero, and R. Vilar, Transformations induced in bulk amorphous silica by ultrafast laser direct writing, Opt. Lett. 38, 4950 (2013). 